\title{
INTEGRAL DOMAINS HAVING NONZERO ELEMENTS WITH INFINITELY MANY PRIME DIVISORS
}

\author{
JIM COYKENDALL AND TIBERIU DUMITRESCU
}

\begin{abstract}
In a factorial domain every nonzero element has only finitely many prime divisors. We study integral domains having nonzero elements with infinitely many prime divisors.
\end{abstract}

Let $D$ be an integral domain. It is well known that if $D$ is a UFD then every nonzero element has only finitely many prime divisors (see e.g. $[\mathrm{G}]$ ). This is also true if $D$ is a Noetherian domain, or more generally, if $D$ satisfies the ascending chain condition for the principal ideals (ACCP). Indeed, if some nonzero element $d \in D$ has infinitely many (mutually non-associate) primes $p_{n}$, then the principal ideals $d / p_{1} \cdots p_{n} D$ form a strictly ascending chain. Moreover, the element $d$ cannot be written as a product of irreducible elements, say $d=a_{1} a_{2} \cdots a_{m}$, because then each $p_{n}$ is an associate of some $a_{j}$, a contradiction; so $D$ is not even an atomic domain. We call a domain having nonzero elements with infinitely many prime divisors an $I P D$ domain. Examples of IPD domains are not hard to find. For instance, the ring $E$ of entire functions, that is, complex functions which are analytic in the whole plane, is an IPD domain. Indeed, by [G, page 147, Exercises 16-21], if $a, b$ are distinct complex numbers, then $z-a, z-b$ are non-associated prime elements of $E$ and if $\left(a_{n}\right)$ is an infinite sequence of distinct complex numbers with $\left|a_{n}\right| \rightarrow \infty$, there exists $0 \neq f \in E$ divisible by each $z-a_{n}$. The subring $A$ of $\mathbf{Q}[X]$ consisting of all polynomials with constant term in $\mathbf{Z}$ is an IPD domain as well, because every prime $p \in \mathbf{Z}$ is prime in $A$ and $p$ divides $X$ (see also Proposition 2). The IPD domains appear in $[\mathrm{MO}]$ under the name of non-GD(1) domains. In [Co], an example of a non-IPD domain whose integral closure is an IPD domain is given.

In this note, we study some transfer properties for the IPD domains and indicate some constructions producing IPD domains. First, we describe the IPD domains of type $A+X B[X]$ (Proposition 2). Here, when $A \subseteq B$ is an extension of domains, $A+X B[X]$ is the subring of $B[X]$ consisting of all polynomials $f$ whose constant term is in $A$. Then, we investigate the possible connections between $D$ being an IPD domain and its integral closure (or its complete integral closure) being an IPD domain (see Propositions 7, 9 and 11).

Throughout this paper, all rings are commutative and unitary. For basic results and terminology our reference is [G].

Let $A \subseteq B$ be a domain extension and $A+X B[X]$ be the subring of $B[X]$ consisting of all polynomials $f$ whose constant term is in $A$. Our first aim is to describe the IPD domains of type $A+X B[X]$.

2000 Mathematics Subject Classification : 13A15, 13B22, 13F05, 13 G05.

Key words and phrases : integral domain, principal primes. 
Lemma 1. Let $A \subseteq B$ be an extension of domains and $0 \neq p \in A$. Then $p$ is a prime element of $A+X B[X]$ if and only if either

(a) $p$ is a prime element of $A$ and $p B=B$, or

(b) $p$ is a prime element of $B$ and $p B \cap A=p A$.

Proof. Set $D=A+X B[X]$. If $p B=B$, then $D / p D \cong A / p A$ and so $p$ is a prime element of $A$ if and only if it is a prime element of $D$. We will therefore assume that $p B \neq B$. If $(b)$ holds, we get the induced domain extension $A / p A \subseteq B / p B$ and $D / p D=A / p A+X(B / p B)[X]$ and so $p$ is prime in $D$.

Conversely, assume that $p$ is a prime element of $D$. Let $c \in p B \cap A$. Then $c=p b$ for some $b \in B$. Since $c X=p(b X),\left.p\right|_{D} c X$. As $p B \neq B, p$ does not divide $X$ in $D$. So, $\left.p\right|_{D} c$, that is, $b \in A$. Hence $p B \cap A=p A$. Consequently, we get the induced ring extension $A / p A \subseteq B / p B$. It is easy to see that $D / p D=A / p A+X(B / p B)[X]$. The conclusion follows.

We are now able to characterize the IPD domains of type $A+X B[X]$.

Proposition 2. Let $A \subseteq B$ be an extension of domains. Then $A+X B[X]$ is an $I P D$ domain if and only if there exists an infinite sequence of mutually non-associate prime elements $\left(p_{n}\right)$ of $A$ such that either

(a) $p_{n} B=B$ for each $n$, or

(b) $\bigcap_{m} p_{m} B \neq 0$ and, for each $n, p_{n}$ is a principal prime of $B$ and $p_{n} B \cap A=$ $p_{n} A$.

Proof. Set $D=A+X B[X]$. Let $\left(f_{n}\right)$ be an infinite sequence of (mutually nonassociate) prime elements of $D$. If infinitely many $f_{n}$ 's are non-constant polynomials, it is easy to see that $\bigcap_{n} f_{n} D=\bigcap_{n} f_{1} \cdots f_{n} D=0$. So $D$ is an IPD domain if and only if there exists an infinite sequence of nonzero elements $p_{n} \in A$ which are prime in $D$ and $\bigcap_{n} p_{n} D=0$. Note that $\bigcap_{n} p_{n} D=\bigcap_{n} p_{n} A+\int_{n} X\left(p_{n} B\right)[X]$. Hence when $D$ is an IPD domain, Lemma 1 shows that either (a) or (b) holds.

Conversely, assume that either (a) or (b) holds. If (a) holds, then $X \in \bigcap_{n} p_{n} D$. While if $(b)$ holds and $0 \neq b \in \bigcap_{n} p_{n} B$, then $b X \in \bigcap_{n} p_{n} D$. In either case, Lemma 1 shows that $D$ is an IPD domain. When condition $(b)$ holds, then $B$ itself is an IPD domain, but note that $A$ may be not, as the example $\mathbf{Z}+X \mathbf{Q}[X]$ shows.

Note that when condition $(b)$ holds, then $B$ itself is an IPD domain, but note that $A$ may be not, as the example $A=\mathbf{Z}+Y \mathbf{Q}[Y]$ shows.

It is easy to see that the proof above works also when replacing $A+X B[X]$ by $A+\left(X_{1}, \ldots, X_{n}\right) B\left[X_{1}, \ldots, X_{n}\right]$.

Corollary 3. A domain $D$ is IPD if and only if $D[X]$ is IPD.

Corollary 4. Let $A$ be a domain, $L$ the algebraic closure of the quotient field of $A$ and $B$ the integral closure of $A$ in $L$. Then $A+X B[X]$ is a not an IPD domain.

Proof. For every principal prime $p \in A, p B \neq B$ and $B$ has no nonzero prime element. Proposition 2 applies.

Corollary 5. Let $D$ be a domain and $K$ a field containing $D$. Then $D+X K[X]$ is an IPD domain if and only if $D$ has infinitely many prime elements.

Consequently, every field $K$ can be embedded in the two-dimensional IPD Bézout domain $K(Y)+X K(Y)[X]$.

Hereafter, for any domain $A$, let $A^{\prime}$ denote its integral closure. 
Corollary 6. Let $D$ be a domain, $K$ a field containing $D$ and set $A=D+X K[X]$. For suitable choices of $D$ and $K$, there exist examples where:

(a) $A^{\prime}$ is an IPD domain and $A$ is not,

(b) $A$ is an IPD domain and $A^{\prime}$ is not.

Proof. By [AHZ, Theorem 2.7], $A^{\prime}=E+X K[X]$ where $E$ is the integral closure of $D$ in $K$. We apply the preceding corollary as follows.

For $(a)$, we take $K$ the quotient field of $D$. We need a domain $D$ with finitely many prime elements and $D^{\prime}$ having infinitely many prime elements. We can use the domain in [H, Example 1]. That is, we take $D=G_{S}$, where $G=L\left[X_{i}^{2}, X_{i}^{3} ; i \geq 1\right], L$ is a field and $S=G \backslash \bigcup_{i}\left(X_{i}^{2}, X_{i}^{3}\right) G$. It was shown in [H] that $D$ is a one-dimensional Noetherian domain without prime elements and $D^{\prime}$ is a PID with infinitely many primes. In fact $D^{\prime}=H_{S}$ where $H=L\left[X_{i} ; i \geq 1\right]$.

For $(b)$, we take $K=\mathbf{C}$ the field of complex numbers and $D=\mathbf{Z}$ the ring of integers. It is well-known that the integral closure of $\mathbf{Z}$ in $\mathbf{C}$, that is, the ring of all algebraic integers, has no prime element.

In contrast with the polynomial extension case (see Corollary 3), power series rings behave differently. Clearly, if $D$ is an IPD domain, say with the nonzero element $d$ having the infinitely many prime divisors $p_{n}$, then $D[[X]]$ is an IPD domain, because each $p_{n}$ remains prime in $D[[X]]$. But there exist non-IPD domains with IPD power series ring. Indeed, let $V$ be a non-discrete one-dimensional valuation domain. Clearly, $V$ is non-IDP since $V$ has no prime element. By [KP, Theorem 2.1], if $a, b$ are distinct non-units of $V$, then $X-a, X-b$ are non-associatted prime elements of $V[[X]]$. Now, choose infinitely many distinct nonzero nonunits elements $a_{n} \in V$. By the proof of [KP, Theorem 3.8], there exists a nonzero $g \in V[[X]]$ which is divisible by all $X-a_{n}$. Hence $V[[X]]$ is an IPD domain.

The second example in [Co] exhibits a one-dimensional quasi-local domain whose integral closure has infinitely many maximal ideals. We modify it to show there exist one-dimensional quasi-local domains with IPD integral closure.

Proposition 7. Every discrete valuation ring with finite residue field can be embedded in a one-dimensional quasi-local domain whose integral closure is a Bézout almost Dedekind IPD domain.

We make an inductive construction as in section 42 of [G]. Our basic step is the following lemma which is a particular case of [G, Theorem 42.5]. Denote by $D_{L}^{\prime}$ the integral closure of domain $D$ in a bigger field $L$.

Lemma 8. Let $D$ be a PID with finitely many primes, say, $p_{1}, \ldots, p_{n}, q, n \geq 0$, each with finite residue field. There exists a quadratic separable field extension $L$ of $K$ (=the quotient field of $D$ ) such that $p_{1}, \ldots, p_{n}$ are primes of $E=D_{L}^{\prime}$ and $q=q_{1} q_{2}$ for two non-associate primes $q_{1}$, $q_{2}$ of $E$.

We shall say that the application of this lemma outputs $\left(E, p_{1}, \ldots, p_{n}, q_{1}, q_{2}\right)$ from the input data $\left(D, p_{1}, \ldots, p_{n}, q\right)$.

Proof of Proposition \%. Let $D_{0}$ be a DVR with finite residue field $D / q_{0} D$ where $q_{0}$ is the prime element of $D$ (e.g., $\left.D_{0}=\mathbf{Z}_{(2)}\right)$. Let Lemma 8 output $\left(D_{1}, p_{1}, q_{1}\right)$ from input $\left(D_{0}, q_{0}\right)$. Inductively, we let Lemma 8 output $\left(D_{n}, p_{1}, \ldots, p_{n-1}, p_{n}, q_{n}\right)$ from input $\left(D_{n-1}, p_{1}, \ldots, p_{n-1}, q_{n-1}\right)$. Set $D=\cup_{n} D_{n}$. Clearly, $D$ is the integral 
closure of $D_{0}$ in the quotient field $L$ of $D$. Moreover, $D$ is an almost Dedekind domain, hence one-dimensional, cf. [G, Corollary 42.2]. Being an inductive limit of PIDs, $D$ is a Bézout domain. By construction, the nonzero prime ideals of $D$ are $p_{n} D, n \geq 1$, and and $\left(q_{1}, q_{2}, \ldots\right) D$, the last one being clearly not finitely generated. In particular, every nonzero prime ideal contains $q_{0}$. Consequently, $D$ is an IPD domain. Consider now its subring $E=D_{0}+q_{0} D$. As $D$ is an overring of $E, D$ is the integral closure of $E$. To complete the proof, we prove that $E$ is quasilocal. Indeed, let $M$ be a maximal ideal of $E$ and $N$ a maximal ideal of $D$ lying over $M$. As $q_{0} \in N$, we get $q_{0} D \subseteq M$, so $M=q_{0} D$, because $q_{0} D$ is a maximal ideal of $E$. Indeed, $E / q_{0} D \cong D_{0} / q_{0} D_{0}$.

For a domain $D$, let $D^{*}$ (resp. $\bar{D}$ ) denote the complete integral closure (resp. the pseudo integral closure) of $D$. Recall that the pseudo integral closure of $D$ is the ring $\bar{D}=\bigcup\left(I_{v}: I_{v}\right)$, where $I$ ranges over nonzero finitely generated ideals of $D$ and $I_{v}=D:(D: I)$ (for a good reference on the concept of pseudo integrality, see [?]). It is easy to see that $D^{\prime} \subseteq \bar{D} \subseteq D^{*}$. The complete integral closure of an IPD domain can be non-IPD. For instance, it is easy to see that the complete integral closure of $\mathbf{Z}+X \mathbf{C}[X]$ is $\mathbf{C}[X]$. For the Archimedean domain case, we have the following positive result. Recall that a domain $D$ is called an Archimedean domain if $\cap_{n} d^{n} D=0$ for each nonunit $d$ of $D$.

Proposition 9. Let $D$ be an Archimedean domain. If $D$ is an IPD domain, then $\bar{D}$ and $D^{*}$ are IPD.

Proof. Let $E$ denote $\bar{D}$ or $D^{*}$. In [DZ, Corollary 2], it was proved that $E \subseteq D_{p D}$ and $p D_{p D} \cap E=p E$ for each prime element $p$ of $D$. In particular, any prime element $p$ of $D$ is also a prime element of $E$ and $p E \cap D=p D$. So two non-associate primes of $D$ are also non-associate in $E$.

Now, assume that $D$ is an IPD domain. Then there exist infinitely many (mutually non-associate) primes $p_{n}$ of $D$ dividing the same nonzero element $x$. As argued above, the same remains true in $E$. So, $E$ is IPD.

Let $D$ be a one-dimensional domain. It is well-known that $D$ is Archimedean. By [DHLZ, Corollary 2.7], the extension $D \subseteq D^{\prime}$ is $R_{2}$-stable in the sense that whenever $0 \neq a, b \in D$ and $a D \cap b D=a b D$, we have $a D^{\prime} \cap b D^{\prime}=a b D^{\prime}$. By [DZ, Proposition 1], every prime element of $D$ remains prime in $D^{\prime}$. So, the proof of Proposition 9 can be used to show the following result.

Corollary 10. Let $D$ be a one-dimensional domain. If $D$ is an IPD domain, then $D^{\prime}$ is IPD.

As our next result shows, if $E$ is an Archimedean IPD domain of dimension $\geq 2$, $E^{\prime}$ is not necessarily IPD, even if $E^{\prime}$ is a finite $E$-module.

Proposition 11. Every discrete valuation ring with finite residue field can be embedded in a two-dimensional Archimedean IPD domain $E$ whose integral closure $E^{\prime}$ is not IPD and $E^{\prime}$ is a finite E-module.

Proof. Let $D$ be the domain constructed in the proof of Proposition 7 and set $a=q_{0}$. As shown there, $D$ is an almost Dedekind domain and, with one exception, all nonzero prime ideals of $D$ are principal. So $D$ is a one-dimensional domain, 
hence an Archimedean domain. Moreover, $a$ belongs to every nonzero prime ideal. Consequently, the quotient field $K$ of $D$ equals $D[1 / a]$.

We adapt a contruction from [DZ, Proposition 3] which in turn uses a construction idea from [DHLRZ, Example 4.1]. Consider the subring $E$ of $D[X]$, $E=D+X(1-a X) D[X]$. Note that $E$ has the following pull-back description. Let $q: D[X] \rightarrow D \times K$ be the ring homomorphism given by $q(f(X))=(f(0), f(1 / a))$. Clearly, $A=q(D)$ is a subring of $D \times K$ isomorphic to $D$. It is easy to see that $E=q^{-1}(A)=\{f(X) \mid f(0)=f(1 / a)\}$. Clearly, $D[X]$ is an overring of $E$ and $U(D)=U(E)$. It easily follows that $E$ is Archimedean.

Let $p$ be a prime element of $D$. We claim that $p D[X] \cap E=p E$, hence $p$ is a prime element of $E$. Indeed, if $f(X) \in D[X]$ and $p f(X) \in E$, then $p f(0)=p f(1 / a)$, so $f(0)=f(1 / a)$, thus $f(X) \in E$. Hence, $p E \cap D=p D$. As $D$ is an IPD domain, so is $E$.

We describe $E^{\prime}$ (=the integral closure of $E$ ). Since $D$ is integrally closed, $E^{\prime} \subseteq$ $D[X]$. For $f(x) \in D[X], f(X) \in E^{\prime}$ if and only if $q(f(X))=(f(0), f(1 / a))$ is integral over $A$ if and only if $f(1 / a) \in D$. So $E^{\prime}=\{f(X) \in D[X] \mid f(1 / a) \in D\}=$ $D+(1-a X) D[X]$. Note that $E^{\prime}=E[a X]$, so $E^{\prime}$ is a finite $E$-module.

To prove that $E^{\prime}$ is not an IPD domain, it suffices to see it has no nonzero constant (polynomial) prime element. But this is done if we show that every prime element $p$ of $D$ is no longer prime in $E^{\prime}$. To this aim, note that $a X, 1-a X$ and $X(1-a X)$ belong to $E^{\prime}$. Since $p$ divides $a$ in $D, p$ divides $(a X)(1-a X)=$ $a(X(1-a X))$ in $E^{\prime}$. Now $g(X)=(a / p) X$ is not in $E^{\prime}$ because $g(1 / a)=1 / p \notin D$. So $p$ does not divide $a X$ in $E^{\prime}$. Also, $p$ does not divide $1-a X$ in $E^{\prime}$ because $p$ does not divide $1-a X$ in $D[X]$. Consequently, $p$ is not prime in $E^{\prime}$. The domain $E$ is the desired example. Note that $E^{\prime}$, hence $E$, is two-dimensional. Indeed, $E^{\prime}$ and $D[X]$ share the ideal $J=(1-a X) D[X]$ and $J$ is maximal in $D[X]$ because $D[X] / J \cong D[1 / a]=K$. So [Ca, Corollaire 2] applies to give that $\operatorname{dim}\left(E^{\prime}\right)=\max \left(\operatorname{dim}(D[X]), \operatorname{ht}_{D[X]} J+\operatorname{dim}\left(E^{\prime} / J\right)\right)=\max (2,1+1)=2$, because clearly $E^{\prime} / J=D$, and $\operatorname{dim}(D[X])=2$ cf. [G, Proposition 30.14].

Call an extension of domains $A \subseteq B$ an IPD-pair if every intermediate ring $A \subseteq$ $D \subseteq B$ is an IPD domain. Let $D$ be the domain constructed in the proof of Proposition 7. We use notation of the proof of Proposition 7 . Then $D \subseteq D\left[1 / p_{1} \cdots p_{n}\right]$ is an IPD pair. Indeed, since $D$ is a Bézout domain, every intermediate ring has the form $D[1 / a]$ where $a$ is the product of some subset of $p_{1}, \ldots, p_{n}$. In contrast, $D \subseteq D[X]$ is not an IPD-pair, because, as shown in the proof of Proposition 11, the intermediate ring $D+\left(1-q_{0} X\right) D[X]$ is not an IPD domain.

Another large class of examples with a similar flavor can be generated as follows. Let $D$ be a Prüfer IPD domain. Select an element $q \in D$ whose prime divisors form an infinite set $X$, and let $Y$ be an infinite subset of $X$. If we set $E:=\bigcap_{p \in Y} D_{p D}$ then $D \subseteq E$ is an IPD pair. To verify this, we note that if $F$ is a ring between $D$ and $E$ then by [G, Theorem 26.1], the elements of $Y$ are distinct primes of $F$. Clearly they continue to divide $q$.

\section{REFERENCES}

[AAZ] D.D. Anderson, D.F. Anderson and M. Zafrullah, Rings between $D[X]$ and $K[X]$, Houston J. Math. 17 (1991), 109-128.

[AHZ] D.F. Anderson, E.G. Houston and M. Zafrullah, Pseudo-integrality, Canad. Math. Bull. 34 (1991), 15-22. 
[CMZ] D. Costa, J.L. Mott and M. Zafrullah, The construction $D+X D_{S}[X]$, J. Algebra 53 (1978), 423-439.

[Ca] P.-J. Cahen, Couples d'anneaux partageant un ideal, Arch. Math. 51 (1998), 505-514.

[Co] J. Coykendall, GD(1) and GD(2) are not preserved in integral closures, Commutative Rings (Compilation of the first four issues of the International Journal of Commutative Rings), Nova Science Publishers, New York, 2002.

[DHLZ] D. Dobbs, E. Houston, T. Lucas and M. Zafrullah, t-linked overrings and Prüfer vmultiplication domain, Commun. Algebra 17 (1989), 2835-2852.

[DHLRZ] D. Dobbs, E. Houston, T. Lucas, M. Roitman and M. Zafrullah, On t-linked overrings, Commun. Algebra 20 (1992), 1463-1488.

[DZ] T. Dumitrescu and M. Zafrullah, LCM-splitting sets in some ring extensions, Proc. Amer. Math. Soc. 130 (2001), 1639-1644.

[G] R. Gilmer, Multiplicative Ideal Theory. Dekker, New York, 1972.

[H] M. Hochster, Non-openness of loci in Noetherian rings, Duke Math. J. 40 (1973), 215-219.

[KP] B.G. Kang and M.H. Park, A localization of a power series ring over a valuation domain, Pure Appl. Algebra 140 (1999), 107-124.

[MO] P. Malcolmson and F. Okoh, Expansions of prime ideals, Preprint.

(Coykendall) Department of Mathematics, North Dakota State University, Fargo, ND 58105-5075, USA

E-mail address: Jim.Coykendall@ndsu.nodak.edu

(Dumitrescu) Facultatea de Matematica, Universitatea Bucuresti, Str. Academiei 14, Bucharest, RO-010014, Romania

E-mail address: tiberiu@al.math.unibuc.ro 